\title{
Chordae Tendineae Prolapse Indicator
}

National Cancer Institute

\section{Source}

National Cancer Institute. Chordae Tendineae Prolapse Indicator. NCI Thesaurus. Code C127562.

An indication as to whether there is a prolapsed cardiac valve chordae tendineae. 